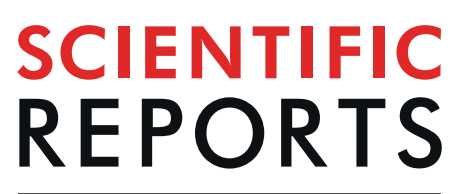

\title{
Survival impact of primary tumor resection in de novo metastatic breast cancer patients (GEICAM/EI Alamo Registry)
}

Sara Lopez-Tarruella $\mathbb{1}^{1,2^{*}}$, M. J. Escudero ${ }^{3}$, Marina Pollan ${ }^{4}$, Miguel Martín $\mathbb{1}^{1,2}$, Carlos Jara ${ }^{5}$, Begoña Bermejo ${ }^{6,2}$, Angel Guerrero-Zotano ${ }^{7}$, José García-Saenz ${ }^{8,2}$, Ana Santaballa ${ }^{9}$, Emilio Alba ${ }^{10,2}$, Raquel Andrés ${ }^{11}$, Purificación Martínez ${ }^{12}$, Lourdes Calvo ${ }^{13}$, Antonio Fernández $\mathbb{1}^{14}$, Norberto Batista ${ }^{15}$, Antonio Llombart-Cussac ${ }^{16}$, Antonio Antón ${ }^{17}$, Ainhara Lahuerta ${ }^{18}$, Juan de la Haba ${ }^{19,2}$, José Manuel López-Vega ${ }^{20}$ \& E. Carrasco ${ }^{3}$

The debate about surgical resection of primary tumor (PT) in de novo metastatic breast cancer (MBC) patients persists. We explored this approach's outcomes in patients included in a retrospective registry, named El Álamo, of breast cancer patients diagnosed in Spain (1990-2001). In this analysis we only included de novo MBC patients, 1415 of whom met the study's criteria. Descriptive, Kaplan-Meier and Cox regression analyses were carried out. Median age was 63.1 years, $49.2 \%$ of patients had singleorgan metastasis (skin/soft tissue [16.3\%], bone [33.8\%], or viscera [48.3\%]). PT surgery (S) was performed in $44.5 \%$ of the cases. S-group patients were younger, had smaller tumors, higher prevalence of bone and oligometastatic disease, and lower prevalence of visceral involvement. With a median follow-up of 23.3 months, overall survival (OS) was 39.6 versus 22.4 months $(H R=0.59, p<0.0001)$ in the $S$ - and non-S groups, respectively. The S-group OS benefit remained statistically and clinically significant regardless of metastatic location, histological type, histological grade, hormone receptor status and tumor size. PT surgery (versus no surgery) was associated with an OS benefit suggesting that loco-regional PT control may be considered in selected MBC patients. Data from randomized controlled trials are of utmost importance to confirm these results.

In western countries metastatic breast cancer $(\mathrm{MBC})$ at first diagnosis is characterized by incidence rates ranging between $4 \%$ and $10 \%$, low rates of long-term survival, and estimates of life expectancy between 18 and 24 months ${ }^{1,2}$. Thus, the consensus management of patients presenting MBC has been palliative, focused on prolonging survival and improving quality of life ${ }^{2}$. Although the impact of new treatment strategies on response rates is clinically meaningful, benefits of new treatments on overall survival (OS) are harder to attain in the metastatic setting ${ }^{3-5}$. Nevertheless, survival gains for MBC patients have been reported ${ }^{6-8}$, suggesting that new therapeutic strategies could potentially improve the clinical practice and management of MBC patients. In

\footnotetext{
${ }^{1}$ Instituto de Investigación Sanitaria Gregorio Marañón, Universidad Complutense, Madrid, Spain. ${ }^{2}$ Centro de Investigación Biomédica en Red de Oncología, CIBERONC-ISCIII, Madrid, Spain. ${ }^{3}$ GEICAM, Spanish Breast Cancer Research Group, Madrid, Spain. ${ }^{4}$ Instituto de Salud Carlos III (ISCIII), Madrid, Spain. ${ }^{5}$ Hospital Universitario Fundación Alcorcón, Universidad Rey Juan Carlos, Madrid, Spain. ${ }^{6} \mathrm{Hospital}$ Clínico Universitario, Valencia. Biomedical Research Institute INCLIVA, Universidad de Valencia, Valencia, Spain. ${ }^{7}$ Instituto Valenciano de Oncología, Valencia, Spain. ${ }^{8}$ Servicio de Oncología Médica, Hospital Clínico San Carlos, Instituto de Investigación Sanitaria del Hospital Clínico San Carlos (IdISSC), Madrid, Spain. ${ }^{9}$ Hospital Universitario La Fe, Valencia, Spain. ${ }^{10}$ Complejo Hospitalario Virgen de la Victoria, Málaga, Spain. ${ }^{11}$ Hospital Universitario Lozano Blesa, Zaragoza, Spain. ${ }^{12}$ Hospital de Basurto, Bilbao, Spain. ${ }^{13}$ Complejo Hospitalario Juan Canalejo, A Coruña, Spain. ${ }^{14}$ Complejo Hospitalario de Albacete, Albacete, Spain. ${ }^{15}$ Hospital Universitario de Canarias, Sta Cruz de Tenerife, Spain. ${ }^{16}$ Hospital Universitario de Lleida Arnau de Vilanova, Lleida, Spain. ${ }^{17}$ Hospital General Universitario Miguel Servet, Zaragoza, Spain. ${ }^{18}$ Instituto Oncológico de Guipúzcoa, San Sebastián, Spain. ${ }^{19}$ Complejo Hospitalario Reina Sofía, Córdoba, Spain. ${ }^{20}$ Hospital Universitario Marqués de Valdecilla, Santander, Spain. *email: sara.lopeztarruella@geicam.org
} 
addition, breakthroughs in diagnostic technology (i.e., PET/TC) now allow the detection of early and asymptomatic metastatic disease. The lower tumor burden in limited stage IV disease makes it potentially more responsive to treatment ${ }^{9-11}$.

Because of these advancements in diagnostics and therapies, clinicians are increasingly acknowledging that MBC patient survival prospects are improving ${ }^{12-14}$ and even challenging the paradigm of incurability in selected cases of de novo $\mathrm{MBC}$ patients ${ }^{15-17}$, particularly those with oligometastatic disease ${ }^{18-20}$. Stemming from the "long-term $\mathrm{MBC}$ survivor profile" concept, the role of local therapies is being re-examined beyond its traditional palliative intent and into a potentially curative one $\mathrm{e}^{21}$.

Retrospective reports and meta-analyses suggest a survival benefit associated with surgery of the primary tumor (PT) in MBC patients, especially when surgical margin is tumor free ${ }^{22-24}$. Thus, surgery may be considered an option in the algorithm for management of selected MBC patients besides the generally accepted hygienic indication as prevention of local complications (infection, ulceration or bleeding). The achievement of locoregional control of the tumor has been tried through surgery but radiotherapy's role has also been studied in this setting ${ }^{25,26}$. Due to the retrospective nature of these studies, reasonable concerns emerged regarding the consistency of the available clinical data ${ }^{27,28}$. Further, recent work, a U.S.-based prospective registry (TBCRC013) and two randomized clinical trials from Turkey and India ${ }^{29-31}$, have reported conflicting results regarding the impact of PT locoregional treatment on OS. Therefore, international guidelines recommend PT surgery only in selected patients while awaiting for the final results of ongoing clinical trials (ECOG 2108/NCT0124800, SUBMIT/ NCT01392586, JCOG 1017/UMIN000005586 y ABCSG 28/POSYTIVE/NCT01015625) ${ }^{32}$

The biological mechanisms underlying the aforementioned results involve the crosstalk among the PT and metastatic foci, PT immunosuppressive effects, and/or the prevention of metastatic re-seeding from the primary source. Yet, the surgical procedure itself may have negative impacts such as a transient inflammation, turning on the "angiogenic switch" in the wound healing process, and a temporary immunosuppressive state which, subsequently, might imbalance microenvironment-tumor interactions eventually triggering the metastatic cascade ${ }^{33-37}$.

The main aim of this work was to study the prognostic value of PT surgical excision, in the presence of other well-known prognostic factors, in MBC patients with OS as the outcome of interest. The objective of the analysis was to clarify the role of a locoregional approach to the PT in de novo MBC patients in a real-world retrospective series. Our ultimate purpose is to evaluate this treatment as a valid option to be included in the national clinical protocol for this patient population. All patients were diagnosed and treated in various Spanish health care institutions included under the umbrella of GEICAM Spanish Breast Cancer Group.

\section{Patients and Methods}

El Álamo study is a cooperative epidemiologic initiative conducted by GEICAM in order to characterize breast cancer (BC) cases diagnosed in Spain. El Álamo is a retrospective registry of patients diagnosed with BC between 1990 and 2001 across 56 Spanish hospitals. The patterns of BC presentation (tumor and host characteristics), the different treatments received, and the clinical evolution of the disease were described and structured in 3 cohorts collected in consecutive 4-year intervals as follows: El Álamo I (1990-1993, 4551 patients, closed by 2000), El Álamo II (1994-1997, 10849 patients, closed by 2003) and El Álamo III (1998-2001, 10675 patients, closed by 2007). This database is estimated to comprise approximately $15 \%$ of the newly diagnosed BC cases in Spain within this period and to reflect clinical practice in the country at each time point. Participating investigators, which included all the medical oncology department staff members of the participating hospitals, were required to include all BC patients seen in those institutions over the entire annual study period.

Only women with invasive de novo MBC were included in the current analysis. Patients with stage I-III at initial diagnosis and later recurrence, with secondary tumors, with fewer than 2 months' follow-up at the same institution, and with missing data regarding their PT surgery were excluded. The criteria to perform PT surgery were defined by institutional guidelines as per investigator discretion.

A total of 39 variables including demographic (age and menopausal status), primary tumor staging (TNM classification), pathology (hormonal receptor status, grade and histological type), and local plus systemic treatment data were selected for this analysis.

Data were collected following the requirements of the Spanish legislation for privacy data protection at the time: the Spanish Organic Law of December $13^{\text {th }}, 1990$ of Personal Data Protection. Observational retrospective studies based on patient charts required neither patient informed consent nor ethical committee approval. We submitted our study to a present-day ethical committee, Hospital Universitario Parc Tauli at Barcelona, which reviewed and confirmed the procedures followed guaranteed patient's data confidentiality and were in compliance with the rules established at that time.

\section{Statistical Analysis}

Categorical variables were expressed as the absolute and relative frequencies. Continuous data were expressed as central tendency and dispersion measures (mean, median, trends, range). Baseline differences in categorical variables were assessed by the Chi Square test $\left(\mathrm{X}^{2}\right)$ or the Fisher exact test when appropriate. OS was defined as the time from the year of diagnosis to death from any cause or to last follow-up visit. OS curves were estimated using the Kaplan-Meier method and survival functions among the different subgroups were compared with the log-rank exact test. The impact of our main variable of interest (PT surgery) and clinically relevant variables (metastatic location, patient's menopausal status, and tumor's characteristics including histological type, histological grade, HER2 status, hormone receptor status, Ki67, expression level of p53, BC subtype, and tumor size) on OS was estimated using Cox proportional hazards regression models. The proportional Hazards assumption was checked using the test based on the correlation coefficient between survival time and the scaled Schoenfeld residuals. All tests were performed using an alpha of 0.05 . 


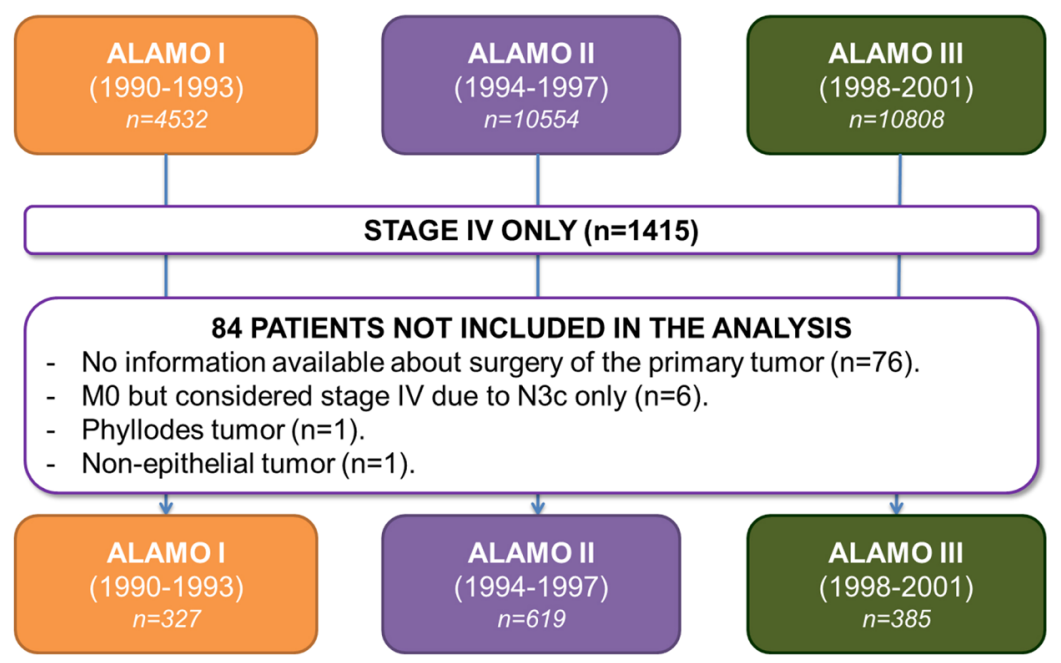

Abbreviations: M, distant metastasis; $\mathrm{N}$ : node; $\mathrm{n}$ : sample size

Figure 1. Patient selection flowchart.

Analyses were performed using the program R and the Statistical Analysis System (SAS) package (Enterprise Guide 5.1 software, SAS Institute Inc., Cary, NC, USA).

\section{Results}

Descriptive analyses. We selected 1415 (5.5\%) patients with de novo MBC from the database El Álamo. Of these, 1331 patients (327 patients from El Álamo I, 619 patients from El Álamo II, and 385 patients from El Álamo $I I I)$ met the eligibility criteria to be included in this analysis (Fig. 1). Table 1 shows patient and tumor characteristics, and treatment received. Median age was 63.1 years (range: $21.6-96.0)$ and $49.2 \%$ of them had single-organ metastasis. The metastatic disease was anatomically distributed as follows: skin/soft tissue (16.3\%), bone (33.8\%), viscera (48.3\%) and unknown/unavailable (8.6\%).

Initial local treatment was the choice for $380(28.5 \%)$ patients (358 surgery and 22 radiotherapy), 722 (54.2\%) patients received initial systemic therapy (480 chemotherapy [CT], 214 endocrine treatment [ET] and 28 both CT and ET), 29 (2.2\%) received best supportive care and for the rest of patients the treatment sequence could not be established (Fig. 2).

PT surgery, at any time of the patient evolution, was performed in $44.5 \%(\mathrm{~N}=592)$ patients (512 radical procedures [86.5\%], 46 palliative procedures [7.8\%], and 34 unknown [5.7\%]). Out of the 592, $427(72.1 \%)$ patients underwent axillary lymph node dissection. Compared to women in the non-surgery group (non-S), women in the surgery group (S) were younger $(19.4 \%$ of the $S$ group versus $11.8 \%$ of the non-S group were $\leq 44$ years-old, respectively), a higher percentage presented with oligometastatic disease (defined as metastases limited to a single organ) than not (57.4\% versus $42.6 \%$, respectively), were more likely to have smaller (i.e. $\leq \mathrm{T} 2)$ tumors $(49.2 \%$ versus $22.7 \%$, respectively), and were less likely to present visceral disease ( $40.3 \%$ versus $54.4 \%$ ) but more likely to present bone metastases (39\% versus $29.8 \%$, respectively).

Survival analysis. With a median follow-up of 23.3 months, median OS was 28.6 months (95\% Confidence Interval [CI], 26.0-31.2). At 1 year, $75.3 \%$ of patients (95\%CI, $72.9-77.6)$ were alive, $42.6 \%$ remained alive at 3 years (95\%CI, 39.8-45.4), and only $25.4 \%$ (95\%CI, 22.8-28) were alive at 5 years (Fig. 3a).

Median OS in the S group was 39.6 months (95\%CI, 35.4-43.9) compared to 22.4 months (95\%CI, 20.7-24.2) in the non-S group (Hazards Ratio [HR] 0.59, 95\%CI, 0.52-0.68; $\mathrm{p}<0.0001)$. At 1 year, $83.6 \%$ of patients $(95 \% \mathrm{CI}$, $80.5-86.6)$ were alive in the $S$ group compared to $68.6 \%(95 \% \mathrm{CI}, 65.2-72.0)$ in the non-S group. This $\mathrm{S}$ group survival advantage was maintained in the following years with $53.7 \%$ of patients (95\%CI, 49.4-57.9) being alive in the S group versus $33.5 \%(95 \% \mathrm{CI}, 29.9-37.2)$ in the non-S group at 3 years. Finally, at 5 years, survivors decreased to $33.5 \%$ of patients $(95 \% \mathrm{CI}, 29.3-37.8)$ in the $\mathrm{S}$ group versus $18.7 \%$ (95\% CI, 15.5-21.9) in the non-S group (Fig. 3b).

Subgroup OS analysis also showed a consistent benefit among the $S$ group across all selected categories of tumor characteristics (Fig. 4).

Univariate analysis for the entire de novo MBC group in the El Álamo registry. PT surgery, metastatic location, histological type, histological grade, hormone receptor status, BC subtype, and tumor size were statistically significant prognostic factors for OS in the univariate Cox analysis. On the other hand, menopausal status $(\mathrm{p}=0.144)$, HER2 status ( $p=0.061), \operatorname{Ki} 67(\mathrm{p}=0.972)$, and $\mathrm{p} 53(\mathrm{p}=0.504)$ failed to have a significant impact on OS (Table 2).

Multivariate analysis for the entire de novo $M B C$ group in the El Álamo registry. Adjusting for all relevant clinical variables in a multivariate model (except BC subtypes and HER2 due to their small sample sizes), the fully-adjusted HR for PT surgery was 0.685 (95\%CI, 0.591-0.793; p < 0.001). All the following variables were 


\begin{tabular}{|c|c|c|c|}
\hline Category & $\begin{array}{l}\text { Surgery } \\
\mathrm{N}=592(\%)\end{array}$ & $\begin{array}{l}\text { Non-Surgery } \\
\mathrm{N}=739(\%)\end{array}$ & p value* \\
\hline Age (years) & & & $<0.0001$ \\
\hline$\leq 44$ & $115(19.4)$ & $87(11.8)$ & \\
\hline $45-64$ & $240(40.5)$ & $283(38.3)$ & \\
\hline$\geq 65$ & $236(39.9)$ & 367 (49.6) & \\
\hline NA & $1(0.2)$ & $2(0.3)$ & \\
\hline Menopausal status & & & 0.0001 \\
\hline Postmenopausal & $419(70.8)$ & $589(79.7)$ & \\
\hline Premenopausal & $168(28.4)$ & $144(19.5)$ & \\
\hline NA & $5(0.8)$ & $6(0.8)$ & \\
\hline Tumor size (TNM) & & & $<0.0001 * *$ \\
\hline T0 & $5(0.8)$ & $5(0.7)$ & \\
\hline $\mathrm{T} 1$ & $63(10.6)$ & $39(5.3)$ & \\
\hline $\mathrm{T} 2$ & $223(37.8)$ & $124(16.8)$ & \\
\hline $\mathrm{T} 3$ & $60(10.1)$ & $84(11.4)$ & \\
\hline $\mathrm{T} 4$ & $212(35.8)$ & $372(50.2)$ & \\
\hline $\mathrm{NA}$ & $29(4.9)$ & $115(15.6)$ & \\
\hline Histological type & & & $<0.0001^{* * *}$ \\
\hline Ductal & $426(72)$ & $322(43.6)$ & \\
\hline Lobular & $42(7.1)$ & $48(6.5)$ & \\
\hline Mucinous (colloid) & $5(0.8)$ & $2(0.3)$ & \\
\hline Mixed & $8(1.4)$ & $2(0.3)$ & \\
\hline Tubular & $2(0.3)$ & $2(0.3)$ & \\
\hline Medullary & $1(0.2)$ & $1(0.1)$ & \\
\hline Papillary & - & $1(0.1)$ & \\
\hline Adenocarcinoma (NS) & $16(2.7)$ & $72(9.7)$ & \\
\hline Others (non-adenocarcinoma or NA) & $92(15.5)$ & $289(39.1)$ & \\
\hline Histological grade & & & 0.2734 \\
\hline GI & $31(5.2)$ & $20(2.7)$ & \\
\hline GII & $163(27.5)$ & $63(8.5)$ & \\
\hline GIII & $138(23.3)$ & $57(7.7)$ & \\
\hline NA & $260(44)$ & $599(81.1)$ & \\
\hline Hormone Receptor status & & & 0.4216 \\
\hline Positive & $286(48.3)$ & $180(24.4)$ & \\
\hline Negative & $102(17.2)$ & $55(7.4)$ & \\
\hline NA & $204(34.5)$ & $504(68.2)$ & \\
\hline HER2 status & & & 0.1243 \\
\hline Positive & $30(5.1)$ & $22(3.0)$ & \\
\hline Negative & $100(16.9)$ & $44(6.0)$ & \\
\hline NA & $462(78.0)$ & $673(91.0)$ & \\
\hline Ki67 (cut-off 13\%) & & & 0.3309 \\
\hline High $(>13 \%)$ & $24(4.1)$ & $13(1.8)$ & \\
\hline Low $(\leq 13 \%)$ & $14(2.4)$ & $4(0.5)$ & \\
\hline NA & $554(93.5)$ & $722(97.7)$ & \\
\hline p53 & & & 0.4370 \\
\hline Positive & $25(4.2)$ & $7(0.9)$ & \\
\hline Negative & $28(4.7)$ & $12(1.6)$ & \\
\hline NA & $539(91.1)$ & $720(97.5)$ & \\
\hline BC Subtypes & & & 0.0758 \\
\hline HR positive/HER2 negative & $76(12.8)$ & $27(3.7)$ & \\
\hline HER2positive & $30(5.1)$ & $22(3)$ & \\
\hline Triple Negative & $18(3.0)$ & $5(0.7)$ & \\
\hline NA & $468(79.1)$ & $685(92.6)$ & \\
\hline Number of organs with metastasis & & & $<0.0001$ \\
\hline 1 & $340(57.3)$ & $315(42.6)$ & \\
\hline 2 & $100(16.9)$ & $170(23.0)$ & \\
\hline$\geq 3$ & $76(12.9)$ & $196(26.5)$ & \\
\hline \multicolumn{4}{|l|}{ Continued } \\
\hline
\end{tabular}




\begin{tabular}{|l|l|l|l|}
\hline Category & $\begin{array}{l}\text { Surgery } \\
\text { N=592(\%) }\end{array}$ & $\begin{array}{l}\text { Non-Surgery } \\
\mathbf{N}=739(\%)\end{array}$ & p value* \\
\hline NA & $76(12.9)$ & $58(7.9)$ & \\
\hline Metastatic location & $\mathrm{N}=528$ & $\mathrm{~N}=689$ & $<\mathbf{0 . 0 0 0 1}$ \\
\hline Skin/Soft tissue & $96(18.2)$ & $102(14.8)$ & \\
\hline Bone & $206(39.0)$ & $205(29.8)$ & \\
\hline Visceral & $213(40.3)$ & $375(54.4)$ & \\
\hline NA & $13(2.5)$ & $7(1.0)$ & \\
\hline
\end{tabular}

Table 1. Patient characteristics by surgical management of primary tumor for the entire group de novo MBC patients from El Álamo registry. Abbreviations: MBC, metastatic breast cancer; NA, not assessed; T, tumor size; NS, non-specific; G, grade; BC, breast cancer; HR, hormone receptor; HER2, human epidermal growth factor receptor 2 . *p-value calculated not considering NA or Other data; **Patients with T0 and T1 tumors were combined; $* * *$ ductal versus others.

\section{De Novo Metastatic BC Alamo I-III $(\mathrm{N}=1331)^{\star}$}

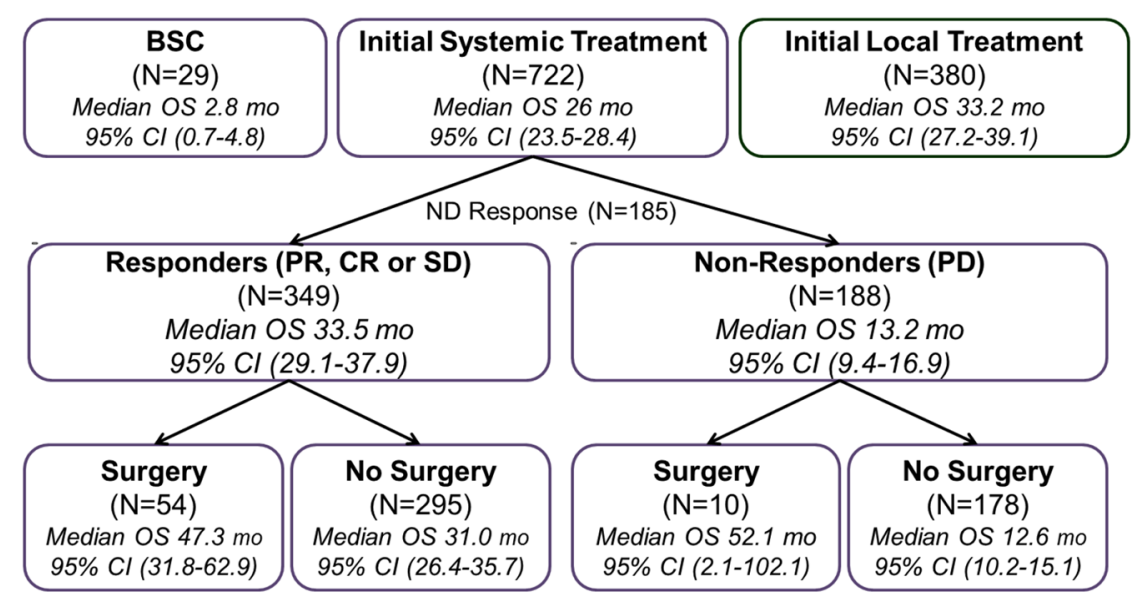

*Treatment sequence could not be established in 200 patients

Abbreviations: MBC, metastatic breast cancer; BC, breast cancer; N, sample size; BSC, best supportive care; OS, overall survival; mo, months; Cl: confidence interval; PR, partial response; $\mathrm{CR}$, complete response; $\mathrm{SD}$, stable disease; $\mathrm{PD}$, progressive disease;

Figure 2. Local and systemic treatments administered to de novo MBC patients and corresponding survival outcomes from El Álamo registry.

found to be statistically significant in the final multivariate Cox regression analysis: metastatic location, histological type, histological grade, hormone receptor status and tumor size (Table 3). Given that HRs for metastatic location and histological type did not meet the proportional hazard assumption, we carried out a sensitivity analysis stratifying the cohort according to these two factors. The HR for PT surgery was virtually unchanged (0.685 in the final model versus 0.684 in the stratified model).

Restricted multivariate analysis (low risk). Due to the observational nature of the El Álamo study and in order to avoid a possible confusion due to indication bias, we performed an exploratory sensitivity analysis for patients with extreme favorable prognostic features. The definition of this "low risk" population comprised patients with small tumor size $(\leq \mathrm{T} 2)$ and oligometastatic disease. We repeated the multivariate Cox regression analysis in this particular subgroup of "low risk" de novo MBC patients $(\mathrm{N}=252)$.

The median OS for the low-risk subgroup was 40.4 months (95\%CI, 33.2-47.7) with 53.2\% (95\%CI, 46.7$59.7 \%$ ) of patients being alive at 3 years. The fully adjusted HR for PT surgery was 0.742 (95\%CI, 0.518-1.063; $\mathrm{p}=0.103)$, i.e., it failed to be a prognostic factor for this low-risk subgroup. However, metastatic location, histological type, and histological grade remained statistically significant predictors of survival. In addition, year of diagnosis (i.e., El Álamo cohort) was also a significant prognostic factor in this patient subgroup.

Subgroup analysis based on the approach for the primary tumor. Table 4 shows a subgroup analysis including metastatic location, histological type, histological grade, hormone receptor status, El Álamo cohort, BC subtype, tumor size, and number of metastases. Median OS was greater in patients with PT surgical excision regardless of 
a. Overall Survival for all de novo MBC patients;

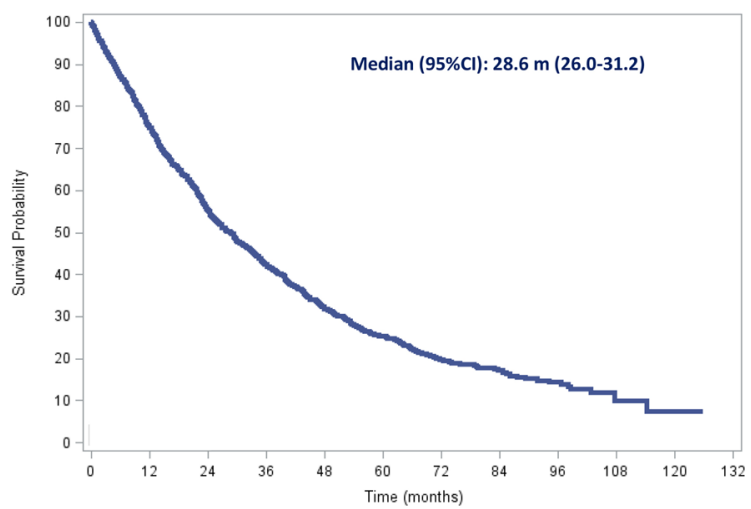

At Risk, $n$

$\begin{array}{llllllllll}1316 & 924 & 640 & 452 & 322 & 223 & 138 & 78 & 32 & 5\end{array}$

Abbreviations: $\mathrm{MBC}$, metastatic breast cancer

b. Overall survival by surgery and no-surgery groups of the primary tumor.

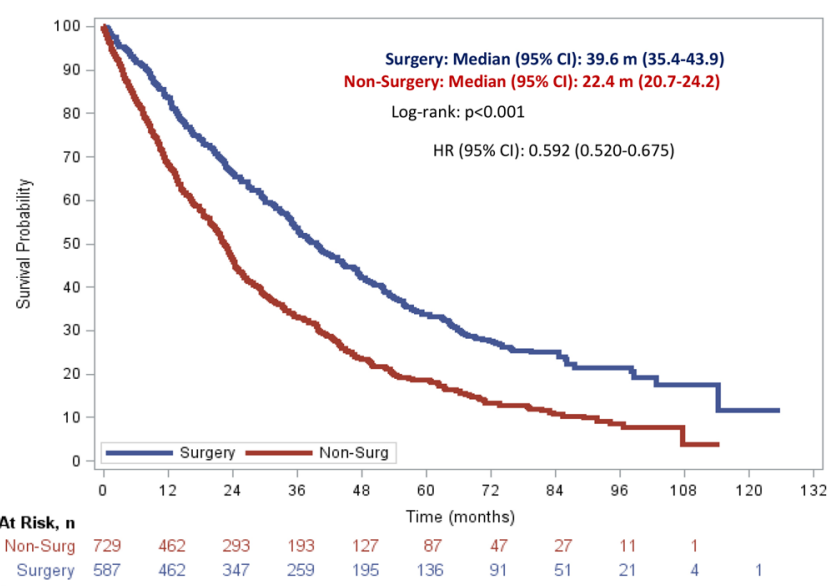

Figure 3. Overall survival for El Álamo registry patients. (a) Overall Survival for all de novo metastatic breast cancer patients; (b) Overall survival by surgery and no-surgery groups of the primary tumor.

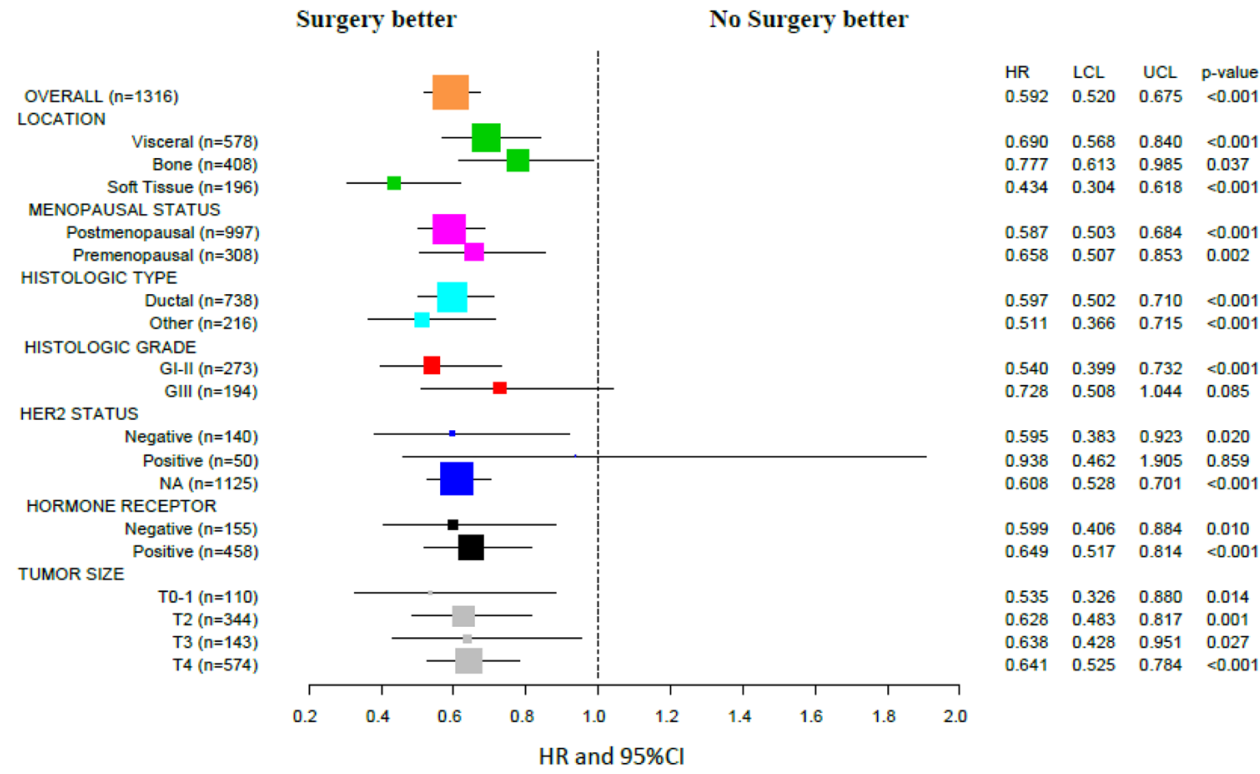

Abbreviations: G, grade; HER2, human epidermal growth factor receptor 2; NA, not-assessed; HR, Hazard ratio; LCL, lower confidence interval limit; UCL, upper confidence interval limit; $T$, tumor; $\mathrm{Cl}$, confidence interval.

Figure 4. Subgroup analysis of overall survival from El Álamo registry. 


\begin{tabular}{|c|c|c|c|c|c|}
\hline Variable & $\mathbf{N}$ & Events & Censored & Hazard Ratio $(95 \% \mathrm{CI})$ & p-value \\
\hline Surgery & & & & & $<0,0001$ \\
\hline Yes & 587 & 384 & 203 & $0.592(0.520-0.675)$ & \\
\hline No & 729 & 560 & 169 & Ref. & \\
\hline Metastatic location & & & & & $<0.0001$ \\
\hline Visceral & 578 & 455 & 123 & Ref. & \\
\hline Bone (if non visceral) & 408 & 276 & 132 & $0.606(0.521-0.704)$ & $<0.0001$ \\
\hline Soft tissue (if non visceral non bone) & 196 & 129 & 67 & $0.568(0.466-0.691)$ & $<0.0001$ \\
\hline Menopausal status & & & & & 0.144 \\
\hline Premenopausal & 308 & 230 & 78 & Ref. & \\
\hline Postmenopausal & 997 & 707 & 290 & $1.117(0.963-1.297)$ & \\
\hline Histological type & & & & & 0.006 \\
\hline Ductal & 738 & 516 & 222 & Ref. & \\
\hline Other & 216 & 163 & 53 & $1.282(1.074-1.529)$ & \\
\hline Histological grade & & & & & 0.001 \\
\hline G I-II & 273 & 184 & 89 & $0.676(0.542-0.844)$ & \\
\hline G III & 194 & 139 & 55 & Ref. & \\
\hline HER2 status & & & & & 0.061 \\
\hline Positive & 51 & 35 & 16 & Ref. & \\
\hline Negative & 140 & 85 & 55 & $0.685(0.461-1.017)$ & \\
\hline Hormone receptor status & & & & & 0.001 \\
\hline Positive & 459 & 309 & 150 & $0.686(0.550-0.854)$ & \\
\hline Negative & 155 & 108 & 47 & Ref. & \\
\hline Ki67 & & & & & 0.972 \\
\hline $0-13$ & 18 & 13 & 5 & $0.988(0.485-2.009)$ & \\
\hline$>13$ & 36 & 19 & 17 & Ref. & \\
\hline P53 & & & & & 0.504 \\
\hline Positive & 31 & 17 & 14 & Ref. & \\
\hline Negative & 40 & 27 & 13 & $0.811(0.439-1.499)$ & \\
\hline El Álamo cohort & & & & & 0.003 \\
\hline El Álamo I & 327 & 248 & 79 & Ref. & \\
\hline El Álamo II & 611 & 438 & 173 & $0.882(0.755-1.031)$ & \\
\hline El Álamo III & 378 & 258 & 120 & $0.742(0.623-0.884)$ & \\
\hline BC Subtype & & & & & 0.039 \\
\hline $\mathrm{TN}$ & 23 & 12 & 11 & Ref. & \\
\hline HER2positive & 51 & 32 & 16 & $1.523(0.790-2.937)$ & \\
\hline HR positive/HER2 negative & 99 & 57 & 42 & $0.881(0.473-1.642)$ & \\
\hline Tumor size (TNM) & & & & & $<0.0001$ \\
\hline T0 & 10 & 5 & 5 & $0.322(0.133-$ & \\
\hline $\mathrm{T} 1$ & 101 & 58 & 43 & $0.779)$ & \\
\hline $\mathrm{T} 2$ & 344 & 236 & 108 & $0.549(0.417-$ & \\
\hline T3 & 143 & 103 & 40 & $0.723)$ & \\
\hline \multirow[t]{3}{*}{$\mathrm{T} 4$} & 574 & 437 & 137 & $0.740(0.631-0.867)$ & \\
\hline & & & & $0.886(0.715-1.099)$ & \\
\hline & & & & Ref. & \\
\hline Number of metastases & & & & & $<0.0001$ \\
\hline Single-organ metastasis & 650 & 443 & 207 & $0.703(0.615-0.804)$ & \\
\hline Multiple metastases & 532 & 417 & 115 & Ref. & \\
\hline
\end{tabular}

Table 2. Univariate analysis of overall survival for the entire group of de novo MBC patients from El Álamo registry. Abbreviations: MBC, metastatic breast cancer; CI: Confidence interval; G, grade; BC, breast cancer; TN, triple negative; HER2, human epidermal growth factor receptor 2; HR, hormone receptor; T, tumor size.

metastatic location (visceral, bone, skin/soft tissue), histological type, hormone receptor status, El Álamo cohort, tumor size, and whether they had single or multiple metastases. However, this benefit failed to reach statistical significance when evaluating it by BC subtypes (due to small sample sizes) or for patients with histological grade III tumors. 


\begin{tabular}{|l|l|l|l|l|}
\hline Variable & N & Hazard Ratio & $\mathbf{9 5 \% C I}$ & p-value \\
\hline Surgery & & & & $<\mathbf{0 , 0 0 1}$ \\
\hline Yes & 587 & 0.685 & $(0.591-0.793)$ & \\
\hline No & 729 & Ref. & & \\
\hline Metastatic location & & & & $<\mathbf{0 , 0 0 1}$ \\
\hline Visceral & 578 & Ref. & & \\
\hline Bone (if non visceral) & 408 & 0.674 & $(0.578-0.787)$ & \\
\hline Soft tissue (if non bone non visceral) & 196 & 0.554 & $(0.452-0.680)$ & \\
\hline NA & 134 & 0.697 & $(0.547-0.886)$ & \\
\hline Histological type & & & & $\mathbf{0 , 0 0 3}$ \\
\hline Ductal & 738 & Ref. & & \\
\hline Other & 216 & 1.249 & $(1.041-1.498)$ & \\
\hline NA & 362 & 0.863 & $(0.725-1.028)$ & \\
\hline Histological Grade & & & & $\mathbf{0 . 0 0 9}$ \\
\hline G I-II & 273 & 0.721 & $(0.576-0.904)$ & \\
\hline G III & 194 & Ref. & & \\
\hline NA & 849 & 0.755 & $(0.613-0.929)$ & \\
\hline Hormone Receptor status & & & & $<\mathbf{0 . 0 0 1}$ \\
\hline Positive & 459 & 0.676 & $(0.539-0.848)$ & \\
\hline Negative & 155 & Ref. & & \\
\hline NA & 702 & 0.983 & $(0.780-1.241)$ & \\
\hline El Álamo cohort & & & & $\mathbf{0 , 2 1 3}$ \\
\hline El Álamo I & 327 & Ref. & & \\
\hline El Álamo II & 611 & 0.940 & $(0.798-1.108)$ & \\
\hline El Álamo III & 378 & 0.839 & $(0.687-1.025)$ & \\
\hline Tumor Size (TNM) & & & & $\mathbf{0 . 0 4 8}$ \\
\hline T0 & 10 & 0.358 & $(0.147-0.873)$ & \\
\hline T1 & 101 & 0.708 & $(0.534-0.939)$ & \\
\hline T2 & 344 & 0.887 & $(0.753-1.046)$ & \\
\hline T3 & 143 & 0.930 & $(0.748-1.156)$ & \\
\hline T4 & 574 & Ref. & & \\
\hline NA & 144 & 0.967 & $(0.779-1.200)$ & \\
\hline & & & & \\
\hline
\end{tabular}

Table 3. Multivariate analysis for overall survival for the entire de novo MBC patients from El Álamo registry*. Abbreviations: MBC, metastatic breast cancer; CI: confidence interval, NA not-assessed; G, grade; T, tumor size. "Even though the variable "Number of metastases" was statistically significant in the univariate analysis, it was not included in the multivariate model because of a multicollinearity problem with the variable "metastatic location".

\section{Discussion}

The OS benefit observed in de novo MBC patients from El Álamo registry undergoing PT surgery supports previous findings reported by contemporary population-based registries ${ }^{14,38-43}$. Two meta-analyses found that PT surgery in these patients was independently associated with improved survival, HR of $0.63-0.69^{22,23}$, ranging from 0.5 to 0.8 in the individual series. However, since other studies failed to detect any benefit from PT surger ${ }^{44-46}$, findings in this field remain inconclusive. El Álamo data yielded a median OS of 39.6 versus 22.4 months $(\mathrm{HR}=0.59, \mathrm{p}<0.0001)$, for the $\mathrm{S}$ and non-S group, respectively. These figures are comparable to the most favorable data reported by previous studies.

De novo MBC patients represented 5.5\% of the entire BC patient population included in the El Álamo registry. PT surgery was performed in $44.5 \%$ of them. These results align with previously reported population-based evidence from other European registries ${ }^{39,41}$. In El Álamo registry $54.2 \%$ of patients received primary systemic therapy and underwent surgery later on.

Although retrospective studies offer valuable information in a very efficient, cost-effective manner, prospective studies are needed to confirm any findings and advance knowledge. For instance, prospective analyses from a US population multicentre registry ${ }^{29}$ recently showed that patients selected for surgery were more likely to have oligometastatic disease and to receive first-line chemotherapy. Among responders, surgery was not associated with improved survival for any BC subtype. Several clinical trials were designed to prospectively address this finding, but results from the two randomized trials already reported failed to provide a satisfactory answer. A Turkish study (MF07-01) $)^{30}$ requiring randomization to loco-regional therapy (LRT) before systemic therapy, reported an OS benefit from initial surgery (median OS of 46 versus 37 months in the $S$ and non-S groups, respectively [HR 0.66, $\mathrm{p}=0.005]$ ), suggesting a potential benefit mainly in patients with bone-only metastases (supporting our results), in patients with ER positive/HER2 negative tumors, as well as in younger patients. In contrast, the Tata Memorial study ${ }^{31}$ requiring induction systemic therapy prior to randomization, reported no LRT impact on OS in patients 


\begin{tabular}{|c|c|c|c|c|}
\hline \multirow[b]{2}{*}{ Subgroup } & \multicolumn{2}{|c|}{ Number of events/patients (\%) } & \multicolumn{2}{|c|}{ Median OS (months) (95\%CI) } \\
\hline & Non-Surgery & Surgery & Non-Surgery & Surgery \\
\hline All $^{\mathbf{a}}$ & $560 / 729(77)$ & $384 / 587(65)$ & $22.4(20.7-24.2)$ & $39.6(35.4-43.9)$ \\
\hline \multicolumn{5}{|l|}{ Metastatic location } \\
\hline Visceral $^{a}$ & $300 / 367(82)$ & $155 / 211(73)$ & $18.1(14.7-21.4)$ & $27.9(20.8-35.0)$ \\
\hline Bone $^{\mathrm{b}}$ & $140 / 204(69)$ & $136 / 204(67)$ & $30.6(25.2-35.9)$ & $42.5(35.5-49.6)$ \\
\hline Skin and Soft tissue ${ }^{\mathrm{a}}$ & 76/101 (75) & $53 / 95(56)$ & $21.9(18.1-25.7)$ & $52.1(37.5-66.8)$ \\
\hline \multicolumn{5}{|l|}{ Histological type } \\
\hline Ductal $^{\mathrm{a}}$ & $246 / 316(78)$ & $270 / 422(64)$ & $23.4(21.2-25.6)$ & $38.7(33.9-43.6)$ \\
\hline Other $^{\mathrm{a}}$ & $113 / 138(82)$ & $50 / 78(64)$ & $16.0(10.1-22.0)$ & $37.9(19.8-56.0)$ \\
\hline \multicolumn{5}{|l|}{ Histological grade } \\
\hline G I-II $^{\mathrm{a}}$ & $66 / 82(80)$ & $118 / 191(62)$ & $25.0(18.2-31.8)$ & $49.7(40.8-58.6)$ \\
\hline $\mathrm{G} \mathrm{III}^{\mathrm{c}}$ & $43 / 56(77)$ & $96 / 138(70)$ & $21.2(12.5-29.9)$ & $29.4(20.6-38.3)$ \\
\hline \multicolumn{5}{|l|}{ Hormone receptor status } \\
\hline Positive $^{\mathrm{a}}$ & $130 / 176(74)$ & $179 / 283(63)$ & $31.8(26.3-37.4)$ & $46.5(40.6-52.3)$ \\
\hline Negative $^{\mathrm{d}}$ & $44 / 54(81)$ & $64 / 101(63)$ & $20.8(10.6-30.9)$ & $23.6(10.2-36.9)$ \\
\hline \multicolumn{5}{|l|}{ El Álamo cohort } \\
\hline El Álamo I ${ }^{\mathrm{a}}$ & $159 / 203(78)$ & $89 / 124(72)$ & $21.2(17.6-24.8)$ & $35.6(28.1-43.2)$ \\
\hline El Álamo $I^{\mathrm{a}}$ & $275 / 357(77)$ & $163 / 254(64)$ & $21.9(19.6-24.2)$ & $38.0(32.0-44.0)$ \\
\hline El Álamo III ${ }^{\mathrm{e}}$ & $126 / 169(75)$ & $132 / 209(63)$ & $25.3(17.8-32.7)$ & $43.6(35.5-51.7)$ \\
\hline \multicolumn{5}{|l|}{ BC subtype } \\
\hline Triple negative & $5 / 5(100)$ & $7 / 18(39)$ & $40(0-94.0)$ & - \\
\hline HER2 positive (regardless HR status) & $12 / 21(57)$ & $23 / 30(77)$ & $38.3(16.4-60.2)$ & $41.8(29.3-54.3)$ \\
\hline HR positive and HER2 negative & $17 / 25(68)$ & $40 / 74(54)$ & $39.3(26.3-52.4)$ & $52.4(39.9-64.9)$ \\
\hline \multicolumn{5}{|l|}{ Tumor size (TNM) } \\
\hline T0 & $2 / 5(40)$ & $3 / 5(60)$ & - & $59.2(10.7-107.7)$ \\
\hline$T 1^{f}$ & $27 / 38(71)$ & $31 / 63(49)$ & $23.9(16.8-30.9)$ & $64.2(42.4-86.1)$ \\
\hline $\mathrm{T}^{\mathrm{a}}$ & $93 / 123(76)$ & $143 / 221(65)$ & $24.0(19.2-28.7)$ & $40.0(32.4-47.6)$ \\
\hline $\mathrm{T} 3^{\mathrm{g}}$ & $62 / 83(75)$ & $41 / 60(68)$ & $24.2(16.2-32.3)$ & $41.0(20.1-61.9)$ \\
\hline $\mathrm{T} 4^{\mathrm{a}}$ & $291 / 365(80)$ & $146 / 209(70)$ & $21.6(19.2-24.0)$ & $35.1(30.4-39.8)$ \\
\hline \multicolumn{5}{|l|}{ Number of metastases } \\
\hline Single-organ metastasis ${ }^{\mathrm{a}}$ & $228 / 313(73)$ & $215 / 337(64)$ & $24.5(22.1-26.9)$ & $40.4(34.5-46.3)$ \\
\hline Multiple metastases $^{\mathrm{e}}$ & $287 / 358(80)$ & $130 / 174(75)$ & $20.8(17.3-24.2)$ & $32.1(28.2-36.0)$ \\
\hline
\end{tabular}

Table 4. Subgroup analysis of overall survival in the surgery and non-surgery groups from El Álamo registry. Abbreviations: OS, overall survival; G, grade; BC, breast cancer; HER2, human epidermal growth factor receptor 2; HR, Hormone receptor; T, tumor size. ${ }^{\mathrm{a}} \mathrm{p}<0.0001 ;{ }^{\mathrm{b}} \mathrm{p}=0.037 ;{ }^{\mathrm{c}} \mathrm{p}=0.083 ;{ }^{\mathrm{d}} \mathrm{p}=0.009 ;{ }^{\mathrm{e}} \mathrm{p}=0.001$; ${ }^{\mathrm{f}} \mathrm{p}=0.002 ;{ }^{\mathrm{g}} \mathrm{p}=0.026$.

who responded to front-line chemotherapy (median OS of 19.2 versus 20.5 months in the LRT versus non-LRT groups [HR 1.04, $\mathrm{p}=0.79]$ ). Nonetheless, local progression-free survival (PFS) was significantly better in patients who had LRT (HR 0.16, $\mathrm{p}<0.001$ ) in contrast with the significant detriment in distant PFS in patients receiving LRT (HR 1.42, $\mathrm{p}=0.012$ ). No differences in survival were detected in the planned subset analysis. In addition, preliminary results from the ABCSG 28 study ${ }^{47}$ comparing initial PT surgery versus initial systemic treatment in only 90 patients (the study was terminated early due to low accrual) failed to find OS benefit for immediate PT surgery (HR 0.69, $\mathrm{p}=0.267$ ). Two other studies (JCOG1017 and ECOG2108) testing the value of surgery after primary systemic treatment are currently ongoing. Although it is agreed upon that level 1 evidence, e.g., prospective data from these randomized-controlled trials like these, is highly needed to close this long-lasting debate, it remains difficult to obtain in a timely manner. Moreover, the interpretation of their results should be done carefully from a methodological perspective and contextualized with the available body of real-world evidence ${ }^{48}$.

Our results should be interpreted in the context of the study's and registry's limitations. For instance, despite the current evidence of the importance of achieving PT surgery with negative margins for improved outcomes $^{38,39,49}$ this information was not available in El Álamo database. Similarly, the database did not address the highly relevant aspect of the impact on survival of PT local radiation therapy, usually studied as part of a multimodal locoregional approach. Thus, whether there is an actual benefit of radiotherapy by itself in this setting, the optimal dose, and fractionation remain unknown ${ }^{26}$ and, thus, cannot be recommended as a standard procedure.

Additionally, because of the observational nature of our series, we performed a multivariate sensitivity analysis in the "low risk" de novo MBC patient subgroup. By reducing the heterogeneity of both known and unknown prognostic factors potential biases are reduced. Results showed that the prognostic value of PT excision surgery was preserved overall, strongly suggesting that the response of this "low-risk" subgroup of patients to PT surgery is not different from that of the de novo MBC patient population under study. 
Further, certain limitations of our data could confound the association between PT surgery and survival, thus affecting how our results inform decision-making algorithms in clinical practice. Specifically, two sources of potential bias should be recognized as selection biases. First, the stage migration phenomenon defining stage IV MBC (TNM-staging classifications changed between 1990 and 2001 in the definition of N3 supraclavicular nodes versus M1); and second, the non-standardized indication of the local procedure which was conducted on a case by case basis, with no pre-defined criteria, based on the physician's discretion.

Also, the lack of on-site monitoring of data compilation and data quality at the different institutions introduces an additional source of variability. Thus, we consider the amount of missing data for the following variables another study limitation: hormone receptor and HER2 status for the molecular sub-classification of patients, surgical procedures, pathological margin status, and chronological order of administration of loco-regional and systemic therapies.

This study also has important strengths. First, to our knowledge this is the largest series performed in Spain $(\mathrm{N}=1331)$ assessing the role of PT surgery in de novo MBC patients' survival. Second, the wide geographical distribution of cases throughout Spain in the late nineties captures the clinical practices in place at that time to manage the controversial aspect of PT surgery as part of the management of a very small but remarkable group of patients. Clinical practices have changed over the last 10-15 years as new treatments have been added to the therapeutic armamentarium. This progress may limit the translational value of the El Álamo results to current clinical practice, but there are still valuable lessons to be learned from them. To address these limitations El Álamo IV (NCT03210974) registry is currently ongoing covering the 2002-2005 period. Moreover, GEICAM is also running, in a parallel fashion, a multicenter prospective registry in the metastatic disease setting (RegistEM, NCT02819882) which will allow the study of how primary tumors are locally managed in a real clinical setting.

Finally, the prognostic value of PT surgical resection may vary by intrinsic subtype, global burden of disease, and/or characteristics of the de novo MBC patients. The definition of the best timing to perform the local approach remains open in the era of molecularly targeted therapy. This is also the case for the need for radiation therapy, the type of surgery required (with or without axillary resection), or even the possibility of foregoing surgery altogether in favor of full radiation treatment.

\section{Conclusions}

In the El Álamo registry local PT surgery was associated with better OS independently of metastatic location, histological type, histological grade, hormone receptor status, and tumor size. These analyses suggest that LRT of PT should be considered as part of the therapeutic strategy for selected patients with advanced disease. Thus, this strategy must be further investigated in randomized controlled trials for de novo MBC patients to control for potential bias intrinsically associated with retrospective studies. Pre-clinical models and translational correlative studies are needed to understand the biology behind the effect of LRT to the PT in this setting.

Ethical approval and inform consent. Data were collected following the requirements of the Spanish legislation for privacy data protection in the considered period. The specific legislation is the Spanish Organic Law of December the 13th, 1990 of Personal Data Protection.

The specific regulation for observational retrospective studies was developed in Spain in 2009 with the December 16th ORDER SAS/3470/2009, which publishes guidelines on post-authorization observational studies for drugs for human use. The ALAMO study was performed before this legislation was in place so the ethical committees in Spain did not review any observational retrospective study before this legislation was developed.

According to the current regulations for observational retrospective studies in force the informed consent of the subjects is not required since: (1) in the process of data collection retrospectively, a secure dissociation procedure is adopted, ensuring that the information handled in the study does not contain personal data and (2) personal interviews are not required nor are biological samples collected from patients. Each patient receives a number identification for their participation in the study.

\section{Data availability}

Data are available from the corresponding author upon reasonable request.

Received: 17 May 2019; Accepted: 2 December 2019;

Published online: 27 December 2019

\section{References}

1. Norton, L. Metastatic breast cancer. Length and quality of life. N. Engl. J. Med. 325, 1370-1371 (1991).

2. Smith, I. Goals of treatment for patients with metastatic breast cancer. Semin. Oncol. 33, S2-5 (2006).

3. Cardoso, F. et al. Second and subsequent lines of chemotherapy for metastatic breast cancer: what did we learn in the last two decades? Ann. Oncol. 13, 197-207 (2002).

4. O'Shaughnessy, J. Extending survival with chemotherapy in metastatic breast cancer. The Oncologist 10(Suppl 3), 20-29 (2005).

5. Hölzel, D. et al. Survival of de novo stage IV breast cancer patients over three decades. Journal of Cancer Research and Clinical Oncology 143, 509-519 (2017).

6. Giordano, S. H. et al. Is breast cancer survival improving? Cancer 100, 44-52 (2004).

7. Gennari, A., Conte, P., Rosso, R., Orlandini, C. \& Bruzzi, P. Survival of metastatic breast carcinoma patients over a 20-year period: a retrospective analysis based on individual patient data from six consecutive studies. Cancer 104, 1742-1750 (2005).

8. Sundquist, M., Brudin, L. \& Tejler, G. Improved survival in metastatic breast cancer 1985-2016. Breast 31, 46-50 (2017).

9. Cermik, T. F., Mavi, A., Basu, S. \& Alavi, A. Impact of FDG PET on the preoperative staging of newly diagnosed breast cancer. Eur. J. Nucl. Med. Mol. Imaging 35, 475-483 (2008).

10. Groheux, D. et al. 18F-FDG PET/CT in staging patients with locally advanced or inflammatory breast cancer: comparison to conventional staging. J Nucl Med 54, 5-11 (2013). 
11. Di Meglio, A. et al. Time trends in incidence rates and survival of newly diagnosed stage IV breast cancer by tumor histology: a population-based analysis. Breast Cancer Res Treat 157, 587-596 (2016).

12. Ernst, M. F. et al. Trends in the prognosis of patients with primary metastatic breast cancer diagnosed between 1975 and 2002. Breast (Edinburgh, Scotland) 16, 344-351 (2007).

13. Tevaarwerk, A. J. et al. Survival in patients with metastatic recurrent breast cancer after adjuvant chemotherapy: little evidence of improvement over the past 30 years. Cancer 119, 1140-1148 (2013).

14. Thomas, A., Khan, S. A., Chrischilles, E. A. \& Schroeder, M. C. Initial Surgery and Survival in Stage IV Breast Cancer in the United States, 1988-2011. JAMA Surg 151, 424-431 (2016).

15. Pagani, O. et al. International Guidelines for Management of Metastatic Breast Cancer: Can Metastatic Breast Cancer Be Cured? JNCI Journal of the National Cancer Institute 102, 456-463 (2010).

16. Andreopoulou, E. \& Hortobagyi, G. N. Prognostic factors in metastatic breast cancer: successes and challenges toward individualized therapy. J. Clin. Oncol. 26, 3660-3662 (2008).

17. Hortobagyi, G. N. Can we cure limited metastatic breast cancer? J. Clin. Oncol. 20, 620-623 (2002).

18. Tait, C. R., Waterworth, A., Loncaster, J., Horgan, K. \& Dodwell, D. The oligometastatic state in breast cancer: hypothesis or reality. Breast (Edinburgh, Scotland) 14, 87-93 (2005).

19. Di Lascio, S. \& Pagani, O. Oligometastatic breast cancer: a shift from palliative to potentially curative treatment? Breast Care (Basel) 9, 7-14 (2014).

20. Salama, J. K. \& Chmura, S. J. Surgery or ablative radiotherapy for breast cancer oligometastases. Am Soc Clin Oncol Educ Book, e8-15 (2015).

21. Tree, A. C. et al. Stereotactic body radiotherapy for oligometastases. Lancet Oncol 14, e28-37 (2013).

22. Petrelli, F. \& Barni, S. Surgery of primary tumors in stage IV breast cancer: an updated meta-analysis of published studies with metaregression. Med Oncol 29, 3282-3290 (2012).

23. Headon, H., Wazir, U., Kasem, A. \& Mokbel, K. Surgical treatment of the primary tumour improves the overall survival in patients with metastatic breast cancer: A systematic review and meta-analysis. Mol Clin Oncol 4, 863-867 (2016).

24. Lee, T. \& Isaacs, C. Treatment of primary breast tumors in de novo metastatic breast cancer. Clin Adv Hematol Oncol 12, 820-827 (2014).

25. Bourgier, C. et al. Breast radiotherapy as part of loco-regional treatments in stage IV breast cancer patients with oligometastatic disease. Radiotherapy and Oncology: Journal of the European Society for Therapeutic Radiology and Oncology 96, 199-203 (2010).

26. Le Scodan, R. et al. Breast cancer with synchronous metastases: survival impact of exclusive locoregional radiotherapy. J. Clin. Oncol. 27, 1375-1381 (2009).

27. Khan, S. A. Surgery for the intact primary and stage IV breast cancer...lacking "robust evidence". Ann Surg Oncol 20, 2803-2805 (2013).

28. Khan, S. A. Primary Tumor Resection in Stage IV Breast Cancer: Consistent Benefit, or Consistent Bias? Annals of Surgical Oncology 14, 3285-3287 (2007)

29. King, T. A. et al. A prospective analysis of surgery and survival in stage IV breast cancer (TBCRC 013). Journal of Clinical Oncology 34, 1006-1006 (2016).

30. Soran, A. et al. Randomized Trial Comparing Resection of Primary Tumor with No Surgery in Stage IV Breast Cancer at Presentation: Protocol MF07-01. Annals of Surgical Oncology (2018).

31. Badwe, R. et al. Locoregional treatment versus no treatment of the primary tumour in metastatic breast cancer: an open-label randomised controlled trial. The Lancet Oncology 16, 1380-1388 (2015).

32. Cardoso, F. et al. 3rd ESO-ESMO International Consensus Guidelines for Advanced Breast Cancer (ABC 3). Ann Oncol 28, 16-33 (2017).

33. Tohme, S., Simmons, R. L. \& Tsung, A. Surgery for Cancer: A Trigger for Metastases. Cancer Res 77, 1548-1552 (2017).

34. Demicheli, R., Retsky, M. W., Hrushesky, W. J. M., Baum, M. \& Gukas, I. D. The effects of surgery on tumor growth: a century of investigations. Ann. Oncol. 19, 1821-1828 (2008).

35. Danna, E. A. et al. Surgical removal of primary tumor reverses tumor-induced immunosuppression despite the presence of metastatic disease. Cancer Res 64, 2205-2211 (2004).

36. Norton, L. \& Massague, J. Is cancer a disease of self-seeding? Nat Med 12, 875-878 (2006).

37. Chiarella, P., Bruzzo, J., Meiss, R. P. \& Ruggiero, R. A. Concomitant tumor resistance. Cancer Lett. 324, 133-141 (2012).

38. Khan, S. A., Stewart, A. K. \& Morrow, M. Does aggressive local therapy improve survival in metastatic breast cancer? Surgery, 132, 620-626; discussion 626-627 (2002).

39. Rapiti, E. et al. Complete excision of primary breast tumor improves survival of patients with metastatic breast cancer at diagnosis. J. Clin. Oncol. 24, 2743-2749 (2006).

40. Gnerlich, J. et al. Surgical removal of the primary tumor increases overall survival in patients with metastatic breast cancer: analysis of the 1988-2003 SEER data. Annals of Surgical Oncology 14, 2187-2194 (2007).

41. Ruiterkamp, J. et al. Surgical resection of the primary tumour is associated with improved survival in patients with distant metastatic breast cancer at diagnosis. Eur J Surg Oncol 35, 1146-1151 (2009).

42. Blanchard, D. K., Shetty, P. B., Hilsenbeck, S. G. \& Elledge, R. M. Association of surgery with improved survival in stage IV breast cancer patients. Annals of Surgery 247, 732-738 (2008).

43. Yoo, T.-K. et al. Identifying long-term survivors among metastatic breast cancer patients undergoing primary tumor surgery. Breast Cancer Research and Treatment 165, 109-118 (2017).

44. Hazard, H. W. et al. Surgical resection of the primary tumor, chest wall control, and survival in women with metastatic breast cancer. Cancer 113, 2011-2019 (2008).

45. Leung, A. M., Vu, H. N., Nguyen, K. A., Thacker, L. R. \& Bear, H. D. Effects of surgical excision on survival of patients with stage IV breast cancer. J Surg Res 161, 83-88 (2010).

46. Dominici, L. et al. Surgery of the primary tumor does not improve survival in stage IV breast cancer. Breast Cancer Res Treat 129, $459-465$ (2011).

47. Fitzal, F. et al. Primary operation in synchroneous metastasized invasive breast cancer patients: First oncologic outcomes of the prospective randomized phase III ABCSG 28 POSYTIVE trial. Journal of Clinical Oncology 35, 557-557 (2017).

48. Khan, S. A. \& DesJardin, E. S. M. Readdressing the Role of Surgery of the Primary Tumor in de Novo Stage IV Breast Cancer. Cancer Treat. Res. 173, 73-88 (2018).

49. Rao, R. et al. Timing of surgical intervention for the intact primary in stage IV breast cancer patients. Ann Surg Oncol 15, 1696-1702 (2008).

\section{Acknowledgements}

This paper was written on behalf of the GEICAM Spanish Breast Cancer Group. We acknowledge the investigators, site staff, and patients of the participating sites, as well as the GEICAM staff involved, for their collaboration and Hosanna Soler for the English editing. This analysis has not received any external funding. All costs were covered by GEICAM, Spanish Breast Cancer Group. The original ALAMO registry was funded 
by Bristol Myers, Roche Farma, Lilly, Novartis, Glaxosmithkline and Pfizer. Partial results of this study were presented at the 37th Annual San Antonio Breast Cancer Symposium, San Antonio, TX, December 2014 and at XV Simposio SEOM, Madrid, Spain, November 2015.

\section{Author contributions}

Study concepts and study design were performed by S.L.T., E.C., M.E., C.J., M.P., M.M. Data acquisition: S.L.T., M.M., C.J., B.M., A.G.Z., J.A.G.S., A.S., E.A., R.A., P.M., L.C., A.F., N.B., A.L.C., A.A., A.L., J.H., J.M.L.V. Data Quality control and algorithms and statistical analysis were done by M.E. Data analysis and interpretation: S.L.T., E.C., M.E. Manuscript preparation: S.L.T., E.C., M.E. Manuscript editing: E.C., M.E. and a manuscript review was performed by all authors: S.L.T., M.M., M.P., C.J., B.M., A.G., J.A.G.S., A.S., E.A., R.A., P.M., L.C., A.F., N.B., A.L.C., A.A., A.L., J.H., J.M.L.V., E.C.

\section{Competing interests}

Dr. López-Tarruella has consultancy/advisory role for AstraZeneca, Novartis, Roche, Pfizer, Celgene, PierreFabre, Eisai and Lilly. Dr. García-Sáenz has received consultancy/speaker fees from Novartis, Celgene, Eli Lilly, EISAI and Roche. Travel support from Novartis, Roche as well as Pfizer and his institution's research funding from AstraZeneca. Dr. Jara has received speaker fees from Roche, Pfizer, Eisai, Novartis and Kyowa as well as travel support from Roche and Pfizer. Dr. Martínez has received consultancy/speaker fees from Leo Pharma, AstraZeneca, Pfizer, and Tesaro as well as travel support from Roche, Rovi and Boehringer Ingelheim. Dr. Martín has a consultant or advisory role with AstraZeneca, Novartis, Roche-Genentech, Pfizer, Glaxo, PharmaMar, Taiho Oncology and Lilly; he has received honoraria from Pfizer and Lilly; he has received research funding from Novartis and Roche. Dr. Guerrero has received travel support from Pfizer. Dr. Antón has consultant/advisory role for Roche and Bayern. Dr. Carrasco has received travel support from Roche and her institution has received funding from Roche, Novartis, Pfizer, BMS, Celgene, AstraZeneca and MSD. Dr. Llombart has received honoraria from Roche, Lilly, Pfizer and Novartis; he has a consultant/advisory role for Roche; he has received funding from Roche, Eisai, AstraZeneca, Lilly and Pfizer and has stock ownership from MedSIR. The rest of the authors declare no conflicts of interest.

\section{Additional information}

Supplementary information is available for this paper at https://doi.org/10.1038/s41598-019-55765-9.

Correspondence and requests for materials should be addressed to S.L.-T.

Reprints and permissions information is available at www.nature.com/reprints.

Publisher's note Springer Nature remains neutral with regard to jurisdictional claims in published maps and institutional affiliations.

(c) (i) Open Access This article is licensed under a Creative Commons Attribution 4.0 International License, which permits use, sharing, adaptation, distribution and reproduction in any medium or format, as long as you give appropriate credit to the original author(s) and the source, provide a link to the Creative Commons license, and indicate if changes were made. The images or other third party material in this article are included in the article's Creative Commons license, unless indicated otherwise in a credit line to the material. If material is not included in the article's Creative Commons license and your intended use is not permitted by statutory regulation or exceeds the permitted use, you will need to obtain permission directly from the copyright holder. To view a copy of this license, visit http://creativecommons.org/licenses/by/4.0/.

(C) The Author(s) 2019 